\title{
Primäres Rhabdomyosarkom der Lunge als seltene Differenzialdiagnose eines kleinzelligen Bronchialkarzinoms
}

\author{
A. Lüdike ${ }^{1}$ \\ J. Knolle ${ }^{2}$ \\ R. Schön ${ }^{3}$ \\ P. Hinze ${ }^{4}$ \\ H. S. Hofmann ${ }^{5}$ \\ J. Schreiber ${ }^{1}$
}

\section{Primary Pulmonary Rhabdomyosarcoma as a Rare Differenzial Diagnosis of \\ Small Cell Lung Cancer}

\section{Zusammenfassung}

Ein primäres pulmonales Rhabdomyosarkom ist eine Rarität. Die histologische Differenzialdiagnose kann schwierig sein. Bei einer 43-jährigen Patientin, Raucherin (25 pack-years), wurde eine solitäre Hirnmetastase diagnostiziert und enukleiert, die histologisch als typisches kleinzelliges Karzinom imponierte. Biopsien aus einem Tumor im linken Lungenoberlappen bestätigten ein kleinzelliges Karzinom, das trotz Chemotherapie jedoch rasch progredient war. Erneute Biopsien zeigten wieder das Bild eines kleinzelligen malignen Tumors. Aufgrund der immunhistochemischen Befunde wurde ein pleomorphes Rhabdomyosarkom diagnostiziert. Bei superinfiziertem Tumorzerfall und Fehlen weiterer Metastasen erfolgte die Lobektomie des linken Lungenoberlappens. Ein postoperatives Pleuraempyem limitierte die Möglichkeiten einer adjuvanten Therapie. Ein frühes Rezidiv mit pleuralen, pulmonalen, Thoraxwand- und spinalen Metastasen erforderte eine Laminektomie und Exstirpation der spinalen Metastasen, sowie eine Strahlentherapie und eine Chemotherapie mit Iphosphamid und Doxorubicin, was zu einer partiellen Remission für wenige Monate führte. Die Patientin verstarb an dem metastasierenden primären pulmonalen Rhabdomyosarkom der Lunge. Diese seltene Tumorentität wurde initial als kleinzelliges Karzinom fehlgedeutet. Eine Würdigung des atypischen klinischen Verlaufs und ein enger Dialog zwischen Pathologen und Klinikern führten zur korrekten Diagnose.

\section{Abstract}

Primary pulmonary rhabdomyosarcoma is a rare entity and the histological differential diagnosis can be difficult. We report on a 43-year old female patient, smoker (25 pack-years), in whom a large solitary brain metastasis was diagnosed and enucleated. Histological examination revealed a typical small cell carcinoma and histological examination of biopsies obtained from a tumor in the left upper lobe of the lung was compatible with a small cell carcinoma. Despite chemotherapy there was a progressive tumor growth. Bronchial biopsies again showed a small cell tumor, although immunohistochemistry proved it to be a pleomorphic rhabdomyosarcoma. Due to the progressive tumor growth with necrosis and superinfection and a lack of further metastases lobectomy of the left upper lobe was performed, complicated by postoperative pleural empyema, limiting the possibilities of adjuvant therapy. Early relapse occurred with pleural, pulmonary, chest wall and spinal metastases. Laminectomy and extirpation of the spinal metastases, local radiotherapy and chemotherapy with iphosphamide and doxorubicine led to partial remission and clinical improvement for few months only. The patient died from metastatic primary rhabdomyosarcoma of the lung. This rare tumor mimicked small cell lung cancer. Appraisal of the atypical clinical course and a close dialogue between pathologists and clinicians enabled the correct diagnosis.

Institutsangaben

${ }^{1}$ Städtisches Klinikum Dessau, Akademisches Lehrkrankenhaus der Martin-Luther-Universität HalleWittenberg, Klinik für Innere Medizin (Chefarzt: Prof. Dr. M. Plauth), Abteilung für Pneumologie

${ }^{2}$ Städtisches Klinikum Dessau, Akademisches Lehrkrankenhaus der Martin-Luther-Universität Halle-Wittenberg, Institut für Pathologie (Chefarzt: Dr. J. Knolle)

${ }^{3}$ Städtisches Klinikum Dessau, Akademisches Lehrkrankenhaus der Martin-Luther-Universität Halle-Wittenberg, Klinik für Neurochirurgie (Chefarzt. Dr. R. Schön)

${ }^{4}$ Martin-Luther-Universität Halle-Wittenberg, Institut für Pathologie (Direktor: Prof. Dr. S. Hauptmann)

${ }^{5}$ Martin-Luther-Universität Halle-Wittenberg, Klinik für Herz-Thorax-Chirurgie (Direktor: Prof. Dr. R. E. Silber)

Korrespondenzadiresse

PD Dr. med. Jens Schreiber · Städtisches Klinikum Dessau · Klinik für Innere Medizin ·

Abteilung für Pneumologie · 06847 Dessau·E-mail: jens.schreiber@klinikum-dessau.de

Eingang: 24. Februar 2005 · Annahme: 19. Mai 2005

Bibliografie

Pneumologie 2005; 59: 456-460 @ Georg Thieme Verlag KG Stuttgart · New York

DOI 10.1055/s-2005-870945

ISSN 0934-8387 
Die malignen Tumore der Atmungsorgane sind in der überwiegenden Mehrzahl Karzinome. Primäre mesenchymale Neubildungen sind mit einer Häufigkeit $<0,1 \%$ aller malignen pulmonalen Neoplasien selten [1]. Sie werden entsprechend ihrer zellulären Differenzierungsrichtung unterteilt (Tab.1). Rhabdomyosarkome der Lunge weisen eine Differenzierung unreifer, embryonaler Skelettmuskulatur auf und stellen ausgesprochene Raritäten dar, über die bisher vorwiegend kasuistisch berichtet wurde [2-4]. Die Symptomatik wird wie bei den Karzinomen durch ihre Lokalisation und das Tumorstadium bestimmt. Über das klinische Verhalten ist im Vergleich zu den Karzinomen wenig bekannt. Die Prognose ist meist ungünstig.

Die histopathologische Differenzialdiagnose pulmonaler Neoplasien stützt sich primär auf die lichtmikroskopische Untersuchung von H/E-gefärbten Gewebsschnitten, die durch immunhistochemische Untersuchungen ergänzt werden kann. Wir berichten über eine Patientin, bei der auf Grundlage des lichtmikroskopischen Bildes initial ein kleinzelliges Bronchialkarzinom diagnostiziert wurde. Erst eine erneute Evaluierung aufgrund des klinischen Verlaufs unter Einsatz immunhistochemischer und elektronenmikroskopischer Untersuchungsverfahren führte zur korrekten Diagnose eines primären pulmonalen Rhabdomyosarkoms.

\section{Fallbeschreibung}

Eine 43-jährige Patientin in gutem Allgemein- und Ernährungszustand wurde aufgrund von Kopfschmerzen, Schwindel und Gesichtsfeldausfällen in eine neurologische Klinik eingewiesen. Es bestanden keine relevanten Vorerkrankungen, jedoch ein Nikotinabusus von 25 pack-years.

Bei der klinischen Untersuchung war eine rechtseitige, gering inkongruente Hemianopsie mit Aussparung der Macula nachweisbar. Der übrige Status praesens war unauffällig.

In der CT und MRT des Neurokraniums war links okzipital ein solitärer Tumor $(7 \mathrm{~cm} \times 3,5 \mathrm{~cm} \times 3,5 \mathrm{~cm})$ mit soliden und zystischen Anteilen und perifokalem Ödem nachweisbar. Die Elektroenzephalographie zeigte ein $\alpha$-EEG mit einer rechtsokzipitalen und geringen rechts-frontalen Funktionsstörung, jedoch keine Zeichen einer erhöhten cerebralen Anfallsbereitschaft. Es erfolgte die Exstirpation des Hirntumors. Die histologische Untersuchung des Operationspräparats (HE-Färbung) zeigte das Bild eines malignen klein-, rund- und blauzelligen Tumors (Abb.1). Dieser wurde, bei der zum Zeitpunkt der Diagnose mitgeteilten Angabe eines primären Lungentumors mit zerebraler Metastase, als kleinzellig-anaplastisches Karzinom eingeordnet. Daraufhin wurde die Patientin in die Klinik für Innere Medizin verlegt.

Die Röntgenaufnahmen (Abb. 2) und die CT des Thorax zeigten eine Raumforderung im linken Oberlappen in einem Tumorstadium T2N0. Bei der Bronchoskopie war B1/2 links durch exophytisches Tumorwachstum verschlossen. Die histologischen Untersuchungen der Biopsien erfolgten in einem auswärtigen Institut für Pathologie und bestätigten den Befund eines „malignen epi-
Tab. 1 Primäre bronchopulmonale Sarkome

\section{Primäre Sarkome der Lungen}

Leiomyosarkom
Rhabdomyosarkom
Spindelzellsarkom
malignes fibröses Histiozytom
Angiosarkom
Fibrosarkom
malignes Hämangioperizytom
neurogenes Sarkom
synoviales Sarkom
Chondrosarkom
Osteosarkom
Kaposi-Sarkom
Liposarkom

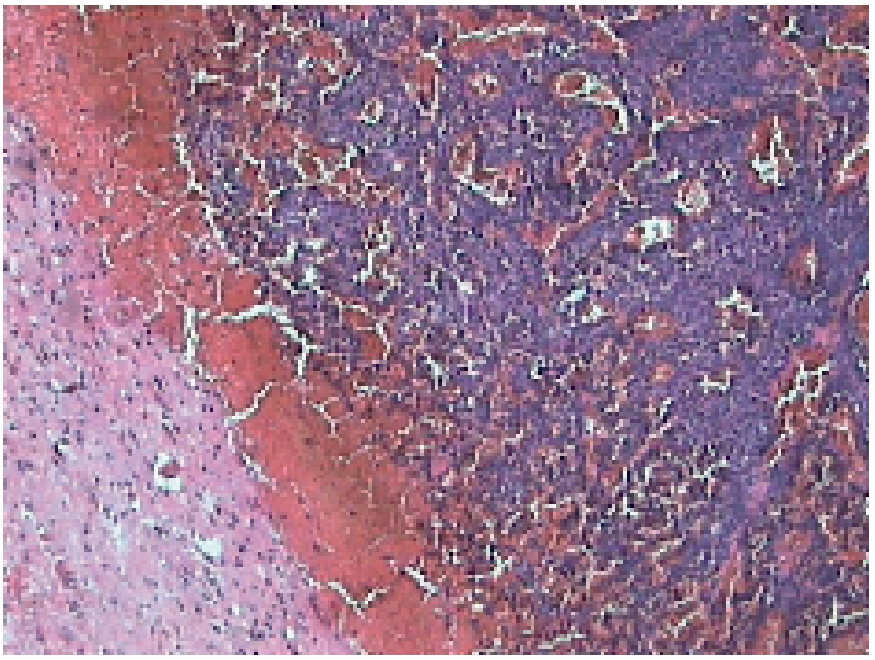

Abb. 1 Zerebrale Metastase eines malignen klein-, rund- und blauzelligen Tumors mit Hämorrhagie (HE 250×).

thelialen Tumors, bestehend aus kleinen haferkornähnlichen Zellen, Oatcell-Karzinom ohne Expression epithelialer Marker, NSE schwach positiv, differenzialdiagnostisch könnte es sich auch um ein undifferenziert wachsendes Plattenepithelkarzinom handeln.“

Die Konzentration des Tumormarkers NSE im Serum war mit $15 \mu \mathrm{g} / \mathrm{l}$ gering erhöht (NB: <12,5 $\mu \mathrm{g} / \mathrm{l}$ ), die Konzentration von CYFRA 21-1 im Normbereich. Skelettszintigraphie, Oberbauchsonographie und abdominelle CT erbrachten keinen Anhalt für weitere Metastasen.

Unter der Diagnose eines kleinzelligen Bronchialkarzinoms vom Oatcell-Typ, extensive disease (T2NOM1 $\left.1_{\text {cer }}\right)$ wurden eine zytostatische Chemotherapie nach dem CEV-Schema und eine Bestrahlung des Neurokraniums durchgeführt.

Bereits nach dem dritten Chemotherapiezyklus musste eine deutliche Progredienz des pulmonalen Tumors konstatiert wer- 


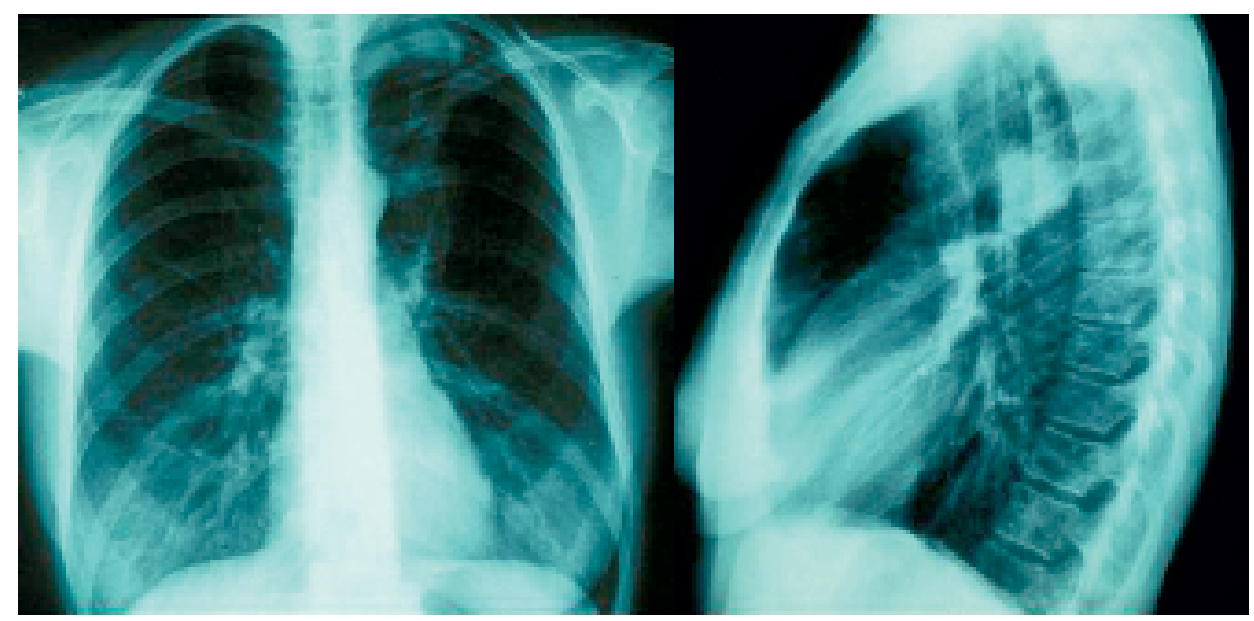

Abb. 2 Röntgenuntersuchung der Thoraxorgane zum Zeitpunkt der Erstdiagnose.

den. Bei einer erneuten Bronchoskopie war der linke Oberlappenbronchus distal des Lingulabronchus durch Tumorgewebe verschlossen. Die histologische Aufarbeitung der Biopsien im Institut für Pathologie des Städtischen Klinikums Dessau erbrachte einen „malignen klein-, rund- und blauzelligen Tumor“ (Abb. 3a). In einigen Bereichen des Tumors waren jedoch auch größere Zellen mit einem breiteren Zytoplasma nachweisbar (Abb. 3b). Aufgrund dieses Befundes wurde im HE-Präparat die Möglichkeit einer rhabdomyosarkomatösen Differenzierung diskutiert und differenzialdiagnostisch neben einem pulmonalen Rhabdomyosarkom eine rhabdomyosarkomatöse Komponente in einem pulmonalen Blastom erwogen. Zusätzliche immunhistochemische Untersuchungen zeigten folgendes Expressionsmuster: Zytokeratin negativ (Abb. 3c), Vimentin positiv, Desmin positiv (Abb. 3d), Aktin negativ, CD56 (N-CAM) positiv, Myoglobin fokal positiv.
Aufgrund dieser Befunde musste die initiale Diagnose eines kleinzelligen Karzinoms in ein Sarkom Grad III mit rhabdomyosarkomatöser Differenzierung revidiert werden. Eine immunhistochemische Reevaluierung der Hirnmetastase und der initialen Bronchusbiopsien bestätigte diese Diagnose.

Aufgrund einer progredienten Zustandsverschlechterung der Patientin infolge eines superinfizierten Tumorzerfalls und nach Ausschluss eines intrakraniellen Rezidivs und sonstiger Fernmetastasen bestand die Indikation zur Lobektomie des linken Oberlappens mit radikaler Lymphadenektomie. In der histomorphologischen Aufarbeitung des Präparats (Institut für Pathologie der Martin-Luther-Universität Halle-Wittenberg) zeigten sich ausgedehnte pulmonale Infiltrate eines pleomorphen Rhabdomyosarkoms mit ausgedehnten Tumornekrosen, Kavernenbildung und schwerer Retentionspneumonie und Pleuritis. Die
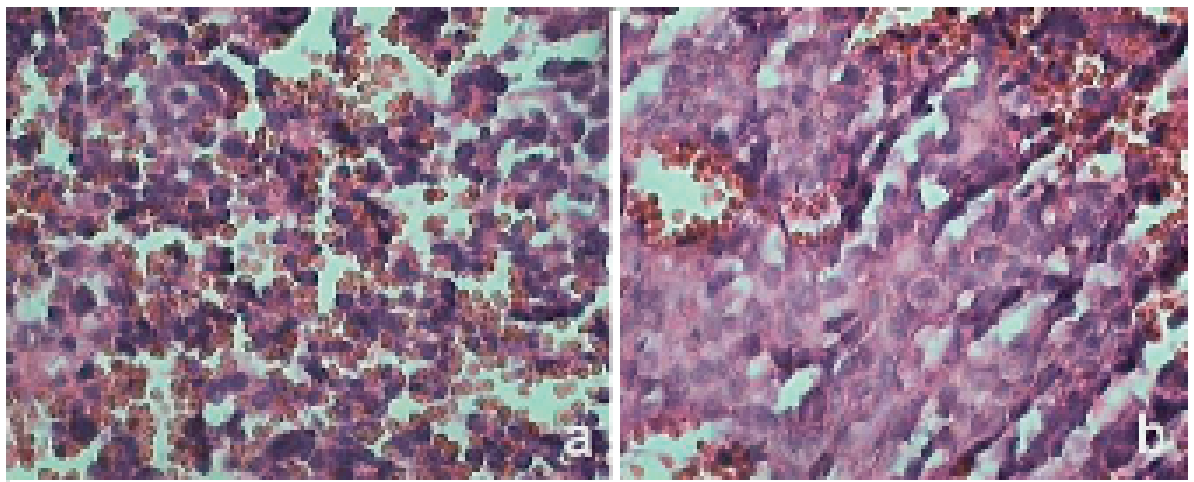

Abb. 3 a Bronchusbiopsie mit massiver Hämorrhagie und Zellen eines malignen klein-, rund- und blauzelligen Tumors (HE $400 \times)$. b Bronchusbiopsie: großzelliger Anteil mit relativ breitem und $z$. T. azidophilem Zytoplasmasaum sowie einzelnen prominenten Nucleoli (HE $400 \times$ ). c Immunhistochemie: Tumorzellen Zytokeratin vollständig negativ (CK $400 \times$ ). d Immunhistochemie: zahlreiche Tumorzellen Desmin positiv (Desmin $400 \times$ ).
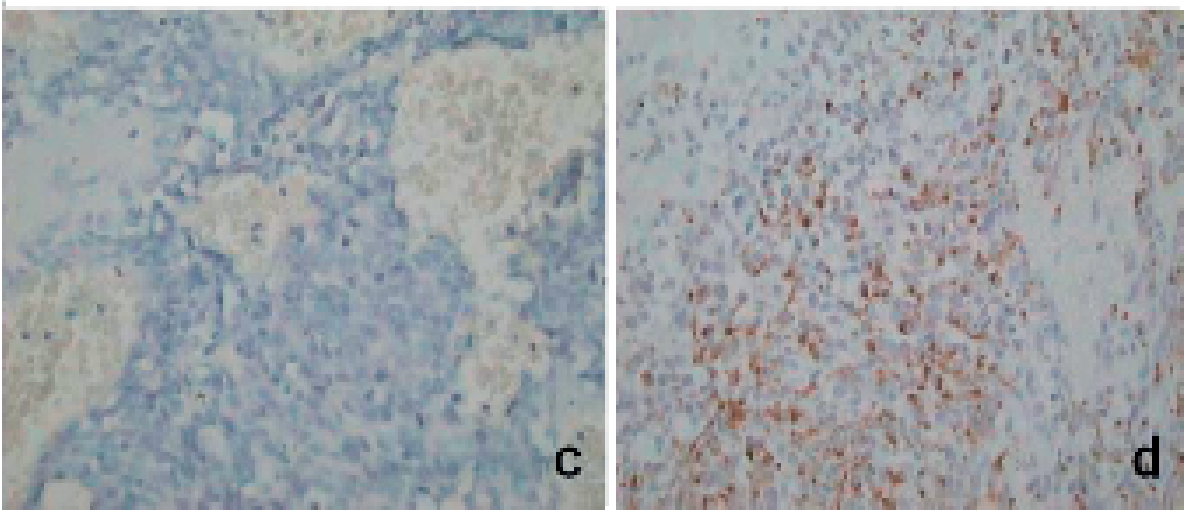
Lymphknoten waren hyperplastisch mit zum Teil eitriger Lymphadenitis und fibrös demarkierten Koagulationsnekrosen. Die Absetzungsränder waren histologisch tumorfrei. Die immunhistochemische und elektronenmikroskopische Untersuchung am Resektat bestätigte die rhabdomyosarkomatöse Differenzierung.

Der postoperative Verlauf wurde durch ein Empyem links und durch eine kontralaterale Pneumonie kompliziert, was die Möglichkeiten einer adjuvanten Therapie limitierte. Nach erfolgreicher Therapie und Ausheilung des Empyems entwickelte die Patientin 3 Monate postoperativ ein inkomplettes Transversalsyndrom mit hochgradiger Paraparese sowie Blasen- und Mastdarmstörungen. Das MRT der Wirbelsäule zeigte eine extraund intraspinale Raumforderung in Höhe der BWK 3 und 4, die das Myelon von dorsolateral links komprimierte. Es lag ein Tumorrezidiv im Bereich des linken Hemithorax vor, das über das Neuroforamen BWK 3/4 per continuitatem nach intraspinal einwuchs. Es wurde notfallmäßig eine spinale Dekompression (Tumorlaminektomie BWK 3/4 und Exstirpation des intraspinalen Tumors) mit adjuvanter Strahlentherapie durchgeführt, was zu einer deutlichen Besserung der neurologischen Symptomatik führte.

Aufgrund der neu aufgetretenen Metastasierung sowohl spinal, als auch pulmonal und im Bereich der Thorakotomienarbe wurde eine Chemotherapie mit Ifosfamid und Doxorubicin durchgeführt, die zu einer partiellen Remission und klinischer Besserung führte, die jedoch nur 4 Monate anhielt. Bei erneutem, rasch progredientem Tumorwachstum verstarb die Patientin an ihrem Grundleiden.

\section{Diskussion}

Das Bronchialkarzinom ist die häufigste maligne Tumorerkrankung beim Mann und zeigt bei Frauen aufgrund veränderter Rauchgewohnheiten eine stetige Zunahme. Voraussetzung für die Entwicklung eines Therapiekonzepts ist die histologische Diagnose, wobei besonders die Differenzierung zwischen kleinzelligen und nicht kleinzelligen Karzinomen relevant ist. Mesenchymale maligne Tumore der Atmungsorgane sind vergleichsweise selten. Ihre Häufigkeit wird mit 0,013-0,05\% aller pulmonalen malignen Tumore angegeben, wobei Rhabdomyosarkome (RMS) in dieser Lokalisation nur kasuistisch beschrieben wurden [1,5-7]. Die Ursache der RMS ist unklar. Diskutiert werden sowohl genetische als auch umweltbedingte Faktoren [8-10].

RMS werden in 3 histologische Typen (National Cancer Institute Classification) eingeteilt [9]. Die embryonale Form wird meist im Kindesalter beobachtet und hat in den letzten Jahren durch multimodale Therapiekonzepte eine deutlich verbesserte Prognose. Die alveoläre Form mit schlechterer Prognose tritt vorwiegend im Kindes- und Jugendalter auf [11]. Die pleomorphe Form kommt vorwiegend bei Erwachsenen, selten jedoch nach dem 40. Lebensjahr vor und hat eine schlechte Prognose [9]. Obwohl RMS prinzipiell in jedem Organ entstehen können, gibt es 3 Prädilektionsstellen: 1. die Kopf-Hals-Region, 2. den Urogenitaltrakt und das Retroperitoneum und 3. die Extremitäten. Andere primäre Lokalisationen, wie die Lungen sind Raritäten $[9,12]$.
Histomorphologisch charakteristisch für das RMS sind spindelzellige Proliferationen mit pleomorphen Abschnitten mit einer wechselnden Anzahl von Rhabdomyoblasten, die an ihrem eosinophilen Zytoplasma bzw. einer selten auftretenden möglichen Querstreifung erkennbar sind [9]. Zusätzlich sind Hämorrhagien und Nekrosen möglich. Die kleinzelligen Anteile können wie in dem hier dargestellten Fall zu Verwechslungen mit einem kleinzelligen Bronchialkarzinom führen. So zeigte sich bei unserer Patientin das Bild eines „malignen klein-, rund- und blauzelligen Tumors“. Dieser deskriptive Terminus beinhaltet lichtmikroskopisch sehr ähnlich erscheinende, histogenetisch jedoch unterschiedliche Tumorentitäten: neben dem kleinzelligen Karzinom und dem RMS auch das Ewing-Sarkom, das periphere Neuroepitheliom, das periphere Neuroblastom, den desmoplastischen klein- und rundzelligen Tumor, Lymphome, das kleinzellige Osteosarkom, das olfaktorische Neuroblastom, das kutane neuroendokrine Karzinom, das kleinzellige Melanom und das mesenchymale Chondrosarkom [13]. Differenzialdiagnostisch sind weiterhin andere pulmonale Sarkome (Tab.1), aber auch sarkomatoide Karzinome, desmoplastische maligne Mesenchymome und reaktive fibrohistiozytäre (inflammatorische) Reaktionen abzugrenzen [5,9]. Kasuistisch wurde über ein RMS, das als akute Leukämie imponierte, berichtet [14]. Deshalb erfordert die histologisch Verifizierung eines RMS neben der Lichtmikroskopie auch auf die Immunhistochemie und in Ausnahmefällen die Elektronenmikroskopie [15].

Zahlreiche immunhistochemische Marker wurden zur Diagnostik der RMS angewandt - ihr diagnostischer Wert, ihre Sensitivität und Spezifität unterscheiden sich jedoch erheblich. Die breiteste Anwendung fanden Antikörper gegen Desmin, Aktin und Myoglobin. Sowohl gegen Desmin als auch Aktin können die immunhistochemischen Reaktionen trotz ihrer hohen Spezifität in undifferenzierten RMS negativ ausfallen. Myoglobin hingegen ist spezifisch, jedoch wenig sensitiv [9]. Aufgrund des Expressionsmusters dieser Antigene darf die Diagnose eines RMS bei unserer Patientin als gesichert betrachtet werden. Bemerkenswert ist jedoch die schwach positive Expression der NSE (neuronenspezifische Enolase). Eine NSE-Positivität wurde in einem geringen Prozentsatz von RMS beschrieben und als Ausdruck neurogener Elemente in diesen Tumoren interpretiert [16 - 21]. Korrespondierend zeigte der Tumor unserer Patientin eine positive Reaktion mit Antikörpern gegen das neuronale Adhäsionsmolekül N-CAM.

Die Diagnose eines primären pulmonalen RMS kann erst nach sorgfältigem Ausschluss einer extrapulmonalen Tumorlokalisation gestellt werden, da die meisten pulmonalen Sarkome Metastasen sind. Bei unserer Patientin lag als einzige initiale extrapulmonale Tumormanifestation eine solitäre Hirnmetastase vor. Es ist nicht mit letzter Sicherheit auszuschließen, dass bei der Patientin ein primäres zerebrales Rhabdomyosarkom mit einer isolierten pulmonalen Metastase vorgelegen hat. Isolierte zerebrale Rhabdomyosarkome wurden sowohl bei Kindern, als auch bei Erwachsenen als extreme Raritäten beschrieben [22,23]. Aufgrund des exophytischen intrabronchialen Tumorwachstums halten wir jedoch einen pulmonalen Primärtumor mit zerebraler Metastasierung für wahrscheinlicher. 
Die Symptomatik pulmonaler Sarkome ist unspezifisch und von der Tumorlokalisation und -stadium abhängig. Die klinische Diagnostik entspricht dem etablierten Vorgehen bei Bronchialkarzinomen, die Stadieneinteilung erfolgt in Analogie zu der TNMKlassifikation der Bronchialkarzinome [6]. Pulmonale Sarkome neigen zur frühzeitigen hämatogenen und lymphogenen Metastasierung $[1,6,12,24]$, so dass zum Diagnosezeitpunkt meist keine kurative Therapie mehr möglich ist. Eine Heilung ist nur durch eine radikale Operation möglich [24] und selbst nach „kurativer" Resektion beträgt die 5-Jahres-Überlebensrate nur ca. $50 \%$ [25]. In lokal fortgeschrittenen oder metastasierten Tumorstadien wird meist ein multimodales Vorgehen angestrebt. Die Prognose ist bei pulmonalen Sarkomen generell, so auch beim adulten pleomorphen RMS sehr ernst. Das mediane Überleben wird mit 7,5 Monaten bis 2 Jahren angegeben. Prognosefaktoren sind Tumorgröße, Grading, Tumorstadium, Operabilität, Tumorlokalisation und Metastasierung [1,5,6,9,24].

Erneut bestätigt der beschriebene Fall, dass gerade bei seltenen Tumorentitäten und schwieriger Differenzialdiagnostik der enge Dialog zwischen Pathologen und Klinikern richtungsweisend sein kann.

\section{Literatur}

${ }^{1}$ Attanoos R, Appleton M, Gibbs A. Primary sarcomas of the lung: a clinicopathological and immunohistochemical study of 14 cases. Histopathology 1996; 29: 29-36

2 Badreddine J, Ledoux J, Marcade E et al. Rhabdomyosarcome pulmonaire primitif: a propos d'une observation. Ann Pathol 2000; 20 : 66-68

${ }^{3}$ Ammar-Boukhris A, Kamoun-Sellami N, Chtourou A et al. Rhabdomyosarcome pulmonaire a presentation endobronchique: a propos d'une observation. Rev Pneumol Clin 2002; 58: 286-289

${ }^{4}$ Ozcan C, Celik A, Ural Z et al. Primary pulmonary rhabdomyosarcoma arising within cystic adenomatoid malformation: a case report and review of the literature. J Pediatr Surg 2001; 36: 1062 - 1065

${ }^{5}$ Keel S, Bacha E, Mark E et al. Primary pulmonary sarcoma: a clinicopathologic study of 26 cases. Mod Pathol 1999; 12: 1124-1131

${ }^{6}$ Regnard J, Icard P, Guibert L et al. Prognostic factors and results after surgical treatment of primary sarcomas of the lung. Ann Thorac Surg 1999; 68: 227-231

${ }^{7}$ Stark P, Eber C, Jacobson F. Primary intrathoracic malignant mesenchymal tumors: pictorial essay. J Thorac Imaging 1994; 9: 148 - 155
${ }^{8}$ Howard G, Casten V. Rhabdomyosarcoma of the orbit in brothers. Arch Ophthalmol 1963; 70: 319-319

${ }^{9}$ Weiss S, Goldblum J. Rhabdomyosarcoma. In: Weiss S, Goldblum J (eds.). Enzinger and Weiss's Soft Tissue Tumors, $4^{\text {th }}$ ed. St. Louis, London, Philadelphia, Sydney, Toronto: Mosby, 2001: 785-836

${ }^{10} \mathrm{Li}$ F, Fraumeni J. Rhabdomyosarcoma in children: epidemiologic study and identification of a family cancer syndrome. J Natl Cancer Inst 1969; 43: 1365 - 1373

${ }^{11}$ Ruymann F, Grovas A. Progress in the diagnosis and treatment of rhabdomyosarcoma and related soft tissue sarcomas. Cancer Invest 2000; 18: $223-241$

12 Przygodzki R, Moran C, Suster S et al. Primary pulmonary rhabdomyosarcomas: a clinicopathologic and immunohistochemical study of three cases. Mod Pathol 1995; 8: $658-661$

${ }^{13}$ Devoe K, Weidner N. Immunohistochemistry of small round-cell tumors. Semin Diagn Pathol 2000; 17: 216-224

${ }^{14}$ Sandberg A, Stone J, Czarnecki L et al. Hematologic masquerade of rhabdomyosarcoma. Am J Hematol 2001; 68: 51-57

${ }^{15}$ Mackay B, Osborne B. The contribution of electron microscopy to the diagnosis of tumors. Pathobiol Annu (United States) 1978; 8: 359-405

${ }^{16}$ Hicks J, Flaitz C. Rhabdomyosarcoma of the head and neck in children. Oral Oncol 2002; 38: 450-459

17 Goji J, Sano K, Murakami R et al. A case of small round cell tumor of the thoracopulmonary region with myogenic and neurogenic elements. Acta Paediatr Jpn 1992; 34: 65 - 71

18 Tsokos M, Linnoila R, Chandra R et al. Neuron-specific enolase in the diagnosis of neuroblastoma and other small, round-cell tumors in children. Hum Pathol 1984; 15: 575-584

${ }^{19}$ Parham D, Webber B, Holt $\mathrm{H}$ et al. Immunohistochemical study of childhood rhabdomyosarcomas and related neoplasms. Results of an Intergroup Rhabdomyosarcoma study project. Cancer 1991; 67: $3072-3080$

${ }^{20}$ Carter R, al-Sams S, Corbett $\mathrm{R}$ et al. A comparative study of immunohistochemical staining for neuron-specific enolase, protein gene product 9.5 and S-100 protein in neuroblastoma, Ewing's sarcoma and other round cell tumours in children. Histopathology 1990; 16: $461-467$

${ }^{21}$ Persson S, Willems J, Kindblom L et al. Alveolar soft part sarcoma. An immunohistochemical, cytologic and electron-microscopic study and a quantitative DNA analysis. Virchows Arch A Pathol Anat Histopathol 1988; 412: 499-513

${ }^{22}$ Matsukado Y, Yokota A, Marubayashi T. Rhabdomyosarcoma of the brain. J Neurosurg 1975; 43: 215-221

${ }^{23}$ Celli P, Cervoni L, Maraglino C. Primary rhabdomyosarcoma of the brain: observations on a case with clinical and radiological evidence of cure. J Neurooncol 1998; 36: 259-267

24 Janssen J, Mulder J, Wagenaar S et al. Primary sarcoma of the lung: a clinical study with long-term follow-up. Ann Thorac Surg 1994; 58: $1151-1155$

${ }^{25}$ Cameron E. Primary sarcoma of the lung. Thorax 1975; 30: 516-520 